Discussion Paper No. 1110

\title{
REACTION TO PRICE CHANGES AND ASPIRATION LEVEL ADJUSTMENTS*
}

\author{
by \\ Itzhak Gilboa ${ }^{+}$ \\ and \\ David Schmeidler ${ }^{++}$
}

June 1994

\footnotetext{
* We are grateful to Enriqueta Aragones for detailed comments on an earlier version of this note.

+ KGSM-MEDS, Northwestern University, Evanston, IL 60208.

+ Department of Statistics, Tel Aviv University and Department of Economics, Ohio State University. Currently visiting the Center for Mathematical Studies, Northwestern University, Evanston, IL 602018.
} 


\begin{abstract}
We claim that preferences of economic agents cannot be assumed given; rather, they are partly determined by the process of trade in the market, by information about the latter and so forth. In other words, preferences determine actions which, in turn, determine preferences. Thus classical tools of analysis such as the neo-classical utility function and the demand curve should be viewed merely as first approximations, which are too simplistic for many purposes.

Changing preferences are not restricted to such phenomena as addiction, advertisement and so forth. Rather, for any product a satisficing consumer has an aspiration level, which is subject to change. The consumer's preferences, as reflected in choice behavior, will also change once the aspiration level is adjusted.

We illustrate these claims by analyzing two examples concerning consumer reaction to price increases. We analyze the effect of aspiration level adjustments on the dynamic pattern of a single consumer's demand, and show that such adjustments generate predictions which do not conform to the neoclassical theory.
\end{abstract}




\section{Introduction}

Main Thesis Economic theory assumes that preferences are given, and based on them market operations can be analyzed. This is a theoretically appealing approach: first identify the fundamentals and based on them study the dynamics, which will hopefully converge to some equilibrium.

In this note we question this two-stage approach. An alternative view suggests that preferences are partly determined by the market process, hence they cannot be assumed a fixed and immutable basis for the analysis of the latter. Rather, consumer preferences are endogenous in that they are affected by the market process, which, in turn, is driven by them. Preferences determine actions, but actions also affect preferences.

Hypothesis Specifically, we focus on one type of change of preferences: we consider a consumer who is "satisficing" rather than optimizing a neo-classical utility function. Such a consumer has an "aspiration level," which, once reached, prevents the consumer from further experimentation. We assume that the aspiration level reflects the consumer's expectations regarding the "consumer surplus;" if it is not attained, the consumer would switch to substitute products.

The key to our analysis is the way the aspiration level is updated over time. The examples analyzed here focus on the effect of past consumption. Other factors which may also effect consumer's aspirations include information about other agents' trades or trade offers, fashion, advertisement and so forth. As for past consumption, we postulate the following rule: the consumer updates her aspiration level (roughly) "towards" the actual consumer surplus she has experienced. Thus past experience changes what the consumer considers to be a "satisfactory" price.

Qualitative Predictions We start by analyzing a consumer's reaction to an increase in a product's price. Section 2 shows that following such an increase, the consumer's demand may fluctuate for a while before she becomes satisficed with a certain consumption level.

We then take the analysis one step further, and show that this hypothesis yields predictions which are at odds with the neo-classical theory. Specifically, in Section 3 we consider the demand for a product, whose price was increased, 
under two scenarios: a sharp, one-shot increase versus a gradual change. We show that our theory predicts that the two scenarios would result in different demand quantities. That is, a gradual change is predicted to result in a larger demand than a corresponding one-shot increase. This stands in contrast to the standard theory, which predicts that the demand would depend solely on the final price.

Finally, Section 4 concludes with several comments.

\section{Non-Monotonic Demand Adjustments}

A consumer is faced with a periodic decision whether to buy a single unit of a certain good. As an example, consider a consumer's daily decision whether to get to work by driving her car or using public transportation, where the good we focus on is gasoline. Thus "buying" the good refers to using one's car, while "passing" means using public transportation. (Of course, one may imagine other consumption decisions which are more directly utility-generating than getting to work.) The decision problem is repeated in the same way, apart from the gasoline price which is subject to change. For simplicity we assume that public transportation cost is fixed, and that the consumer is informed of the gasoline price only after she decided to make a purchase, if indeed she did. The analysis does not depend on this assumption and may be modified to incorporate a consumer's ability to look ahead and imagine her well-being if indeed she decides to buy the product at the new price. Similarly, one can assume that the public transportation cost is also subject to change; however, we would like to focus on a single commodity model to facilitate the comparison with the neoclassical theory.

To model satisficing behavior, we apply case-based decision theory (CBDT). (See Gilboa and Schmeidler (1992).) However, no familiarity with CBDT is assumed here. The choices available to the consumer on each day are $\{$ buy, pass $\}$. The consumer is assumed to have some "intrinsic value" $v$ for driving her own car. (The precise meaning of this parameter will be discussed below.) In case of a purchase decision, the consumer experiences a "consumer surplus" of $(v-p)$ where $p$ is the price the consumer ended up paying for gasoline. If, on the other hand, our consumer ended up using public transportation ("passing"), we assume that the corresponding "consumer surplus" is a fixed parameter $w$, which takes into account the presumably fixed price of that alternative. 
The consumer has an "instantaneous utility" function which depends on the consumer's surplus and on an aspiration level with respect to which the utility is scaled. Denoting the aspiration level at time $t$ by $h^{t}$, the utility function at time $t$ from an outcome $x$ is given by

$$
u^{t}(x)=x-h^{t}
$$

The consumer is maximizing a functional $U$ which computes, for each possible act, a similarity-weighted sum of the utility of the results this act led to in the past. The similarity function here is rather simple: it merely reflects the time that has past since the recalled case. Specifically, an experience which dates back $j$ periods is assumed to be "discounted" by a factor of $\beta^{j}$ (where $\beta \in(0,1)$ ). Discounting of the past attempts to capture both the probability of recall, and the intuition that "old" experiences are deemed less important or less relevant than "new" ones.

The functional $U$ is therefore defined as follows: at time zero, it is identically zero. If at time $(t-1)$ the consumer chooses the act buy, and finds a price $p$, we get

$$
U^{t}(b u y)=\left(v-p-h^{t-1}\right)+\beta U^{t-1}(b u y)
$$

and

$$
U^{\prime}(p a l s s)=\beta U^{t-1}(p a s s)
$$

If, on the other hand, she chooses pass, the functional is given by:

$$
U^{\prime}(b u y)=\beta U^{t-1}(b u y)
$$

and

$$
U^{t}(p a s s)=\left(w-h^{t-1}\right)+\beta U^{t-1}(p c c s s)
$$

Thus our consumer will keep purchasing the product as long as $U^{\prime}(b u y)>U^{\prime}(p a s s)$ and will leave the market once the reverse inequality holds. (The theory does not specify the choice in case of indifference.) Of course, a consumer who decided to "leave the market," namely not to buy the product, may later decide to "come back" and decide to purchase again.

Our next major assumption concerns the consumer's aspiration level. We assume that the aspiration level is updated over time, depending on the 
consumer's experiences. More specifically, suppose our consumer experiences a "consumer surplus" of $x$. Basically, the aspiration level is updated to be some weighted average of its previous value and this evaluation of the new consumption experience. However, we will assume that the aspiration level tends to be somewhat below this value. That is, rather than considering the value $x$, the consumer's aspiration will be adjusted towards the value $(x-\delta)$ for some fixed $\delta>0$.

The intuition behind this assumption is as follows: if the aspiration level tends to the consumer surplus precisely, in the long run the consumer obtains her aspiration level and is just barely satisficed. For such a consumer the smallest shock (such as a minuscule price increase) would render the product unsatisfactory. This does not seem to be entirely plausible. People tend to exhibit some "stickiness" or "inertia" in decisions in general, and purchase decisions in particular. A price change which is smaller than some "just noticeable difference" is unlikely to make all consumers, who are used to buy the product, turn away from it to untried alternatives.

"Inertia" can be modeled and explained in several ways. (See, for instance, Bar-Ilan and Blinder (1992) who discuss "rational inertia.") In terms of aspiration level adjustments, inertia may be viewed as a consumer's tendency to be "content." That is, a decision maker who "gets used" to a given alternative and becomes content with it, will exhibit inertia in her decisions.

Thus we postulate a "contentment factor" $\delta>0$ for each consumer. This number is a measure of the contentment our consumer feels with respect to a product she purchases regularly. In our model, by setting the aspiration level lower than the mere average of past consumption experiences, these experiences become slightly more than just-satisficing.

Formally, the speed of adjustment of the consumer's aspiration level is given by $\alpha \in(0,1)$, and the aspiration level at time $t$, after experiencing a consumer surplus $x$, is

$$
h^{t}=\alpha(x-\delta)^{+}+(1-\alpha) h^{t-1}
$$

where $(y)^{+}=\max \{y, 0\}$. Notice that the new experience - evaluated as $(x-\delta)-$ is not allowed to become negative for the aspiration level update. In particular, if the consumer decided to make a purchase and found a price $p$ which exceeds her "intrinsic value" $v$, her aspiration is updated as if she faced a price $v$ (or $(v-\delta)$ ). 
This means that the consumer will not "learn" to be satisficed with prices exceeding her intrinsic value: the aspiration level will not drop below zero, and any negative consumer surplus will be deemed unsatisfactory regardless of the number of times it has been experienced in the past.

Let us now assume that the sequence of prices $\left(p^{t}\right)_{t}$ is such that $p^{t}=p$ for all $t \geq T$ and some $T \geq 0$. Consider a consumer with $v-\delta \geq p$, that is a consumer whose intrinsic value and contentment factor allow her to be content with the price $p$, and assume that she has indeed been purchasing the product regularly (say, for all $t \geq T$ ). We then get

$$
h^{\prime} \longrightarrow(v-p-\delta)
$$

and

$$
U^{\prime}(b u t y) \underset{t \rightarrow \infty}{\longrightarrow} \frac{\delta}{1-\beta}, \quad \quad U^{t}(p a s s) \underset{t \rightarrow \infty}{\longrightarrow} 0
$$

Notice that, as long as the price does not exceed $(v-\delta)$, the long-run $U$ level of our consumer does not depend on $p$. This follows from our supposition that the aspiration level is adjusted over time and that the consumer "gets used" to a certain price level. This is not the case when one considers "too high" prices, i.e., $p>v-\delta$. To such a price our consumer will not "get used." Hence the "intrinsic value" $v$ can be interpreted precisely thus: it is the highest price (up to $\delta$ ) to which the consumer can possibly adjust.

It should be emphasized that the "intrinsic value" in this model differs from the concept of "private value." While the latter is typically interpreted as the maximal price at which the consumer will decide to make a purchase, in our model the consumer may not buy at lower prices than $v$, and she may decide to buy at higher prices as well. For a price lower than $v$, the consumer may still be unsatisficed if she expected a higher consumer surplus. Specifically, if the aspiration level is significantly above $(v-p)$ for a long enough period, the $U$ value of $b u$. will become negative even though $(v-p)$ may be positive. In this case the consumer will turn away from the product to some alternative. On the other hand, a price which exceeds ' does not prohibit a purchase. While such a price would result in negative $U$ values, the alternative may still be worse ( $U$ wise). Thus the "intrinsic value" in our model does not tell the whole story, and in particular, it certainly does not suffice to predict the consumer's behavior. 
Rather, it should be thought of as the highest price - over various scenarios and consumption histories - with which the consumer can still be satisficed.

Let us consider the effect of a price increase, say from some $p$ to a new value $p^{\prime}$, on the behavior of a consumer characterized by $v, \delta, \alpha$, and $\beta$. Assume that for a relatively long period (where "relatively long" is determined by $\alpha$ and $\beta$ ), the price $p$ has prevailed and the consumer has been purchasing the product. Then we start at

$$
h^{i} \approx l-p-\delta
$$

and

$$
U^{\prime}(b u !) \approx \frac{\delta}{1-\beta} . \quad U^{t}(\text { pass }) \approx 0 .
$$

The new experience yields a "-value of

$$
u^{\prime}\left(v^{\prime}-p^{\prime}\right)=v-p^{\prime}-h^{\prime} \approx \delta-\left(p^{\prime}-p\right)
$$

and

$$
U^{t+1}\left(b u(y) \approx u^{\prime}\left(v-p^{\prime}\right)+\frac{\beta \delta}{1-\beta} .\right.
$$

Thus, if $p^{\prime}-p>\frac{\delta}{1-\beta}$, the consumer will become dissatisfied with the product and will not purchase it in the next period. On the other hand, if the price increase is small enough (so that the reverse inequality holds), the consumer will continue to purchase the product at $p^{\prime}$.

Let us now focus on those consumers who decide to switch to the act pass. They experience

$$
u^{i+1}(u)=u^{-} h^{i+1}
$$

We further distinguish among this subset of consumers. For some, $w-h^{t+1}>0$, and these will be satisficed with the alternative of public transportation. The aspiration level will continue to be updated, so that for large $\tau$ we get $h^{\tau} \approx w-\delta$. That is, these consumers may first be "quite happy" with public transportation and wonder why they haven't chosen it to begin with, but over time they will get used to this wonderful alternative and be "just content" 
with it. At any rate, they will not go back to choosing buy (i.e., driving their own cars) unless further changes occur.

For other consumers we may have $w-h^{i+1}<0$. These consumers have no reason to feel regret over their past choices: indeed, the alternative of public transportation turned out to be worse than using one's car at the old price of gasoline. However, prices being what they are now, they are not satisficed with either alternative. If the aspiration level remains fixed, they will alternate between buy and pass with frequencies determined by $\left(v-p-h^{t}\right),\left(w-h^{t}\right)$, and $\beta$. By contrast, when the aspiration level is updated over time, each consumer will become more-or-less satisficed with at least one of the two options and will settle for either buy or pass. (See related analysis, for the case $\beta=1$, in Gilboa and Schmeidler $(1993 \mathrm{a}, \mathrm{b})$.)

Thus the dynamic pattern we should observe is as follows: some consumers keep buying gasoline, and others switch to public transportation for ever; yet another subset alternates for a while, and eventually splits into two subsets: those who go back to driving their cars (and consuming gasoline), and those who end up using public transportation (and do not buy gasoline). In particular, a consumer's reaction to a price increase need not be monotone with respect to time.

Note that aggregate demand may or may not be a monotonically decreasing function (of time) following a price increase. If, for instance, all consumers are synchronized in their decisions, aggregating over their demand may result in a non-monotonic reaction, predicting an "overshooting" phenomenon: first demand drops sharply, then it goes up again to some intermediate level. However, if the timing of consumption decisions varies across consumers, say due to existing inventory, the aggregation of nonmonotonic functions may still be monotonically decreasing.

\section{Speed of Price Change and Demanded Quantity}

We now wish to take the analysis one step further, and show that our model provides predictions which differ from those of the neo-classical theory. Specifically, we would like to show that in our model a gradual price increase may have a different effect on demand than a corresponding one-shot increase.

Let us assume that for a long period the price of gasoline was fixed at $p_{0}$. We would like to compare two scenarios: (i) a one-shot price increase to $p_{2}>p_{0}$, 
after which the price is held fixed at $p_{2}$; and (ii) a two-stage increase: first to a price level $p_{1}$ such that $p_{2}>p_{1}>p_{0}$, and then, after a long enough period of adjustment, to $p_{2}$.

For any given price increase, it is obvious that there may be consumers for which it is small enough to keep purchasing gasoline, and others for which it is not. The main point is that those consumers who remain in the market get used to the new price (provided it does not exceed their "intrinsic value") in the long run, and after a long enough period their $U$-value for buying tends back to the same value $\frac{\delta}{1-\beta}$. For such consumers a similar price increase in the future will have similar effects. In other words, any given consumer will remain in the market as long as (i) the current price does not exceed her "intrinsic value"; and (ii) the price increases along the path are small enough.

Coming back to the comparison of the two scenarios above, one can find ranges of the parameters $v, \delta$, and $\beta$ for which the increase from $p_{0}$ to $p_{2}$ is "too large", while both increases from $p_{0}$ to $p_{1}$ and from the latter to $p_{2}$ are not. Consumers whose parameters are in those ranges will keep purchasing the product in scenario (i) but would refrain from doing so in (ii).

Thus the question, "How many consumers will decide to buy at price $p_{2}$ ?" is ill-posed. In our model, it has no unique answer since the final price $p_{2}$ simply does not contain all the relevant information. Correspondingly, one cannot assume a demand curve, which may be viewed as a collection of answers to such questions. Consumer preferences - as reflected in economic behavior depend on the economic process and cannot be assumed given independently of it.

\section{Concluding Remarks}

Changing Preferences The adjustment of aspiration level may be viewed as a special case of changing preferences. Indeed, the literature provides generalizations of the neo-classical theory which can deal with phenomena involving changes in tastes, such as in cases of addiction, aging, advertisement and the like. (This literature dates back to Allais (1947) and Strotz (1956), and includes more recent contributions such as Pollak (1968, 1970 and 1976) and Becker $(1991 a, b)$.) But we claim that the change of preferences is pervasive and ubiquitous enough to undermine the basic tenets of the neo-classical theory. One 
may incorporate the phenomena we discuss here in generalizations of the neoclassical framework, for instance by assuming that the neo-classical utility function is defined over consumption streams. But we suspect that such generalizations would result in a theory which is almost tautologically true and thus useless.

Reference Theory Kahneman and Tversky have shown in experimental works (see Kahneman and Tversky (1984)) that consumer preferences may depend on the consumer's current consumption bundle. They have also suggested a new consumer theory, according to which the consumer's "neo-classical" utility function is parametrized by this bundle. (Tversky and Kahneman (1990).)

In spirit, our theory bears some resemblance to theirs. However, we allow preferences to depend on the whole history of consumption, and highlight the role of aspiration level adjustments.

The Supply Side The focus of this paper is consumer's behavior and the underlying theory from which one derives the concept of a "demand curve." Assuming that consumers can be "satisficed", and that their aspiration level is subject to change, we claim that the demand curve cannot be assumed given, or independent of the market process.

While the supply side is typically assumed to involve "more rational" agents than the consumers on the demand side, there is no reason to believe that the supply curve is a fixed characteristic of the market either. First, in many markets - such as the market for used cars, real-estate and so forth - the supply is also determined by individual economic households, which are not "more neoclassical" than the buyers. Second, even when firms, rather than households, constitute most of the supply side, their behavior may follow satisficing models more accurately than profit-maximization models. Wherever there are satisficing agents involved, there is room for aspiration level update, and preferences become intertwined with the economic process they are supposed to govern. 


\section{References}

Allais, M. (1947), Economie et Interet. Paris, Imprimerie Nationale.

Bar-Ilan, A. and A. S. Blinder (1992), "Consumer Durables: Evidence on the Optimality of Usually Doing Nothing," Lournal of Money, Credit and Banking, 24: 258-272.

Becker, G. S. (1991a), "Rational Addiction and the Effect of Price on Consumption," American Economic Review Papers and Proceedingsis 81 no. 2: 237-241.

Becker, G.S. (1991b), "Habits, Addiction and Traditions." Nancy L. Schwartz Lecture, Northwestern University.

Gilbor, I. and D. Schmeidler (1992), "Case-Based Decision Theory." Working paper, Northwestern University. Revised, 1993.

Gilbod, I. and D. Schmeidler (1993a), "Case-Based Consumer Theory." Working paper, Northwestern University.

Gilboa, I. and D. Schmeidler (1993b), "Case-Based Optimization." Working paper, Northwestern University.

Kahneman, D. and A. Tversky (1984), "Choices, Values and Frames." American Psychologist 39: $341-350$.

Pollak, R. A. (1968), "Consistent Planning." Review of Economic Studies 35: 201-208.

Pollak, R. A. (1970), "Habit Formation and Dynamic Demand Functions." Lournal of Political Esonomy 78: 745-764.

Pollak, R. A. (1976), "Habit Formation and Long-Run Utility Functions." Lournal of Economic Theory 13: 272-297.

Strotz, R. H. (1956), "Myopia and Inconsistency in Dynamic Utility Maximization." Review of Econumic Studies 23: 165-180).

Trersky, A. and D. Kahneman (199(1), "Reference Theory of Choice and Exchange." Working paper. 